\title{
Azabicycloalkenes as Synthetic Intermediates - Application to the Preparation of Diazabicycloalkane Scaffolds
}

\author{
Alexander H. G. P. Prenzel, Nina Deppermann and Wolfgang Maison* \\ Universität Hamburg, Institut für Organische Chemie, \\ Martin-Luther-King-Platz 6, 20146 Hamburg
}

General Remarks: Melting points were determined in open capillaries in a Dr. Lindström instrument and are uncorrected. - ${ }^{1} \mathrm{H}-\mathrm{NMR}$ and ${ }^{13} \mathrm{C}-\mathrm{NMR}$ spectra were recorded with a Bruker-Karlsruhe AMX 400 spectrometer $(400 \mathrm{MHz} / 100.6 \mathrm{MHz})$ or on a Bruker-Karlsruhe DRX 5001 spectrometer (500 MHz / $125.8 \mathrm{MHz})$. Chemical shifts, $\delta$, are presented in part per million (ppm) and coupling constants, $J$, in Hertz $(\mathrm{Hz})$ from tetramethylsilane (TMS, $0 \mathrm{ppm}$ ) as the internal standard for $\mathrm{CDCl}_{3}$ and residual solvent peaks for other deuterated solvents. - Mass spectra were obtained with a Varian MS MAT 311A in El mode, a VG/70-250 F (VG Analytical) instrument in FAB mode in a $p$-nitrobenzylalkohol matrix. - Elemental analyses were performed with a C, H, N-Analyser EA 1108 from Carlo Erba. - The following educts were synthesized according to literature procedures: aminals $\mathbf{5}^{1}$, Cbz-protected bromoglycinate $6^{2}$ and azabicycloalkene $16^{3}$. - Solid supported piperidine was purchased from Aldrich and washed under nitrogen atmosphere three times with abs THF before use.

\footnotetext{
${ }^{1}$ Harayama, Y.; Yoshida, M.; Kamimura, D.; Kita, Y. Chem. Commun. 2005, 17641766.

${ }^{2}$ Williams, R. M.; Aldous, D. J.; Aldous, S. C. J. Org. Chem. 1990, 55, 4657-4663.

${ }^{3}$ Lautens, M.; Fagnou, K.; Zunic, V. Org. Lett. 2002, 4, 3465-3468. 

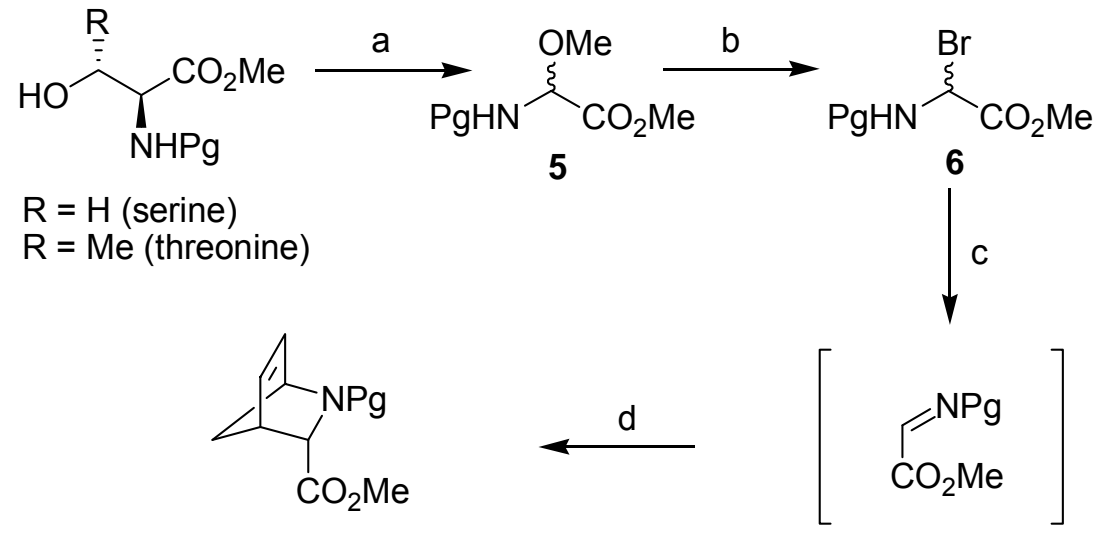

S-8a, $\mathrm{Pg}=\mathrm{Fmoc}, 73 \%$ yield, dr: $18: 1,64 \%$ ee purity $70-90 \%$ S-8b, Pg = Cbz, $70 \%$ yield, dr: 18:1, $72 \%$ ee

Reagents and conditions: ${ }^{a} \mathrm{C}_{6} \mathrm{~F}_{5} \mathrm{I}\left(\mathrm{OCOCF}_{3}\right)_{2}, \mathrm{MeCN}, \mathrm{MeOH}, 1 \mathrm{~h}, \mathrm{rt} ;{ }^{\mathrm{b}} \mathrm{PBr}_{3}, \mathrm{CCl}_{4}, 24 \mathrm{~h}$, rt; ${ }^{\mathrm{c}}$ piperidine on solid support, $\mathrm{CH}_{2} \mathrm{Cl}_{2}, 20 \mathrm{~min}$, rt; ${ }^{\mathrm{d}}$ cyclopentadiene, $10 \mathrm{Mol} \%$ TolBINAP-CuClO${ }_{4}, \mathrm{CH}_{2} \mathrm{Cl}_{2},-78{ }^{\circ} \mathrm{C}$, $2 \mathrm{~h}$.

\section{Fmoc-Bromide 6:}

Under an atmosphere of nitrogen $2.72 \mathrm{~g}$ (7.97 mmol) Fmoc-protected aminal 5 was dissolved in $200 \mathrm{ml}$ anhydrous $\mathrm{CCl}_{4}$ and $8.63 \mathrm{~g}(31.87 \mathrm{mmol})$ freshly distilled $\mathrm{PBr}_{3}$ was added. The solution was stirred for $72 \mathrm{~h}$ and the solvent was evaporated in vacuo. The residue was suspended in dry pentane. Filtration under nitrogen atmosphere yielded a colourless solid which was used in the next step without further purification; ${ }^{1} \mathrm{H}-\mathrm{NMR}\left(400 \mathrm{MHz}, \mathrm{CDCl}_{3}\right): \delta 7.31-7.78(\mathrm{~m}, 8 \mathrm{H}), 6.36(\mathrm{~d}, 1 \mathrm{H}, \mathrm{J}=$ $10.2 \mathrm{~Hz}), 6.16(\mathrm{~d}, 1 \mathrm{H}, \mathrm{J}=9.40 \mathrm{~Hz}), 4.50$ (s, 2H), 4.24 (s, 1H), 3.89 (s, 3H); ${ }^{13} \mathrm{C}-\mathrm{NMR}$ $\left(\mathrm{CDCl}_{3}, 101 \mathrm{MHz}\right): \delta 143.6,141.7,138.2,129.4,128.6,128.3,127.5,125.6,125.6$, $125.3,120.5,54.1,52.9,47.2,21.8$.

\section{General procedure for the preparation of carbamate protected azabicycloalkenes (3S)-8:}

Under an atmosphere of nitrogen $16 \mathrm{mg}(0.05 \mathrm{mmol})$ of $\mathrm{CuClO}_{4} \cdot 4 \mathrm{MeCN}$ and $37 \mathrm{mg}$ $(0.055 \mathrm{mmol})(R)$-tolBinap were dried in vacuo at $50{ }^{\circ} \mathrm{C}$ using a water bath. After $1 \mathrm{~h}$ $0.5 \mathrm{ml}$ abs. dichloromethane were added and the catalyst solution was stirred for 30 $\min$.

A second flask was charged with $0.5 \mathrm{mmol}$ of bromide 6 in $2 \mathrm{ml}$ abs. dichloromethane. Polymer bound piperidine ( $1 \mathrm{mmol}$ piperidine) was added and the suspension was stirred at room temperature for $20 \mathrm{~min}$. The resulting solution was transferred with a syringe to the catalyst solution through a Nagelne ${ }^{\circledR}$ Teflon 
membrane filter. The flask was sealed with Parafilm ${ }^{\circledR}$ and then cooled to $-78{ }^{\circ} \mathrm{C}$. Freshly distilled cyclopentadiene $(1 \mathrm{mmol})$ was added slowly and the solution was stirred at $-78{ }^{\circ} \mathrm{C}$ for another $2 \mathrm{~h}$. After warming to room temperature, the solution was filtered through silica and the solvent was removed in vacuo. The product was purified by column chromatography on silica.

\section{Azabicycloalkene (3S)-8a}

According to the general procedure for the preparation of carbamate protected azabicycloalkenes $0.16 \mathrm{~g}(0.41 \mathrm{mmol})$ of bromide 6 was converted to $0.11 \mathrm{~g}$ of azabicycloalkene (3S)-8a using $10 \mathrm{Mol} \%(R)$-tolBinap and $\mathrm{CuClO}_{4} \cdot 4 \mathrm{MeCN}$ as catalyst $(73 \%) ; R_{\mathrm{f}}=0.34$ (PE / EtOAc 2:1; UV); ${ }^{1} \mathrm{H}-\mathrm{NMR}\left(400 \mathrm{MHz}, \mathrm{CDCl}_{3}, 1: 1\right.$ rotameric mixture): $\delta 7.30-7.54(\mathrm{~m}, 9 \mathrm{H}), 6.16-6.50(\mathrm{~m}, 2 \mathrm{H}), 4.85(\mathrm{br} \mathrm{s}, 0.5 \mathrm{H}), 5.62$ (br s, 0.5H), $4.45(\mathrm{~d}, 1.5 \mathrm{H}, \mathrm{J}=6.0 \mathrm{~Hz}), 4.25-4.30(\mathrm{~m}, 1 \mathrm{H}), 4.16-4.17(\mathrm{~m}, 0.5 \mathrm{H})$, $3.77(\mathrm{~s}, 1.5 \mathrm{H}), 3.73(\mathrm{~s}, 1.5 \mathrm{H}), 3.54$ (br s, 0.5H), 3.42 (br s, 0.5H), 3.33 (br s, 1H), 1.95 - $2.00(\mathrm{~m}, 1 \mathrm{H}), 1.50(\mathrm{~d}, 1 \mathrm{H}, \mathrm{J}=8.7 \mathrm{~Hz}) ;{ }^{13} \mathrm{C}-\mathrm{NMR}\left(\mathrm{CDCl}_{3}, 101 \mathrm{MHz}\right.$, mixture of rotamers): $\delta 141.7,137.6,136.7,128.0,125.3,120.3,67.5,67.4,62.1,61.7,60.7$, 59.4, 59.2, 52.8, 49.5, 48.5, 47.6, 46.1, 45.4; HRMS (ESI): calcd. for $\mathrm{C}_{23} \mathrm{H}_{21} \mathrm{NO}_{4}$ $\left(\mathrm{MH}^{+}\right)$: 375.1471; found: 375.1578; HPLC (CHIRALPAK ${ }^{\circledR}$ AD-H $(5 \mu \mathrm{m})$, Hexan: isoPropanol 85:15, flow $0.5 \mathrm{ml} / \mathrm{min}, \lambda=210 \mathrm{~nm}$ ): ee $=64 \%$; $t_{\mathrm{R}}$ (major) $=31.3 \mathrm{~min} ; t_{\mathrm{R}}$ $($ minor $)=37.8 \mathrm{~min}$.

\section{Azabicycloalkene (3S)-8b}

According to the general procedure for the preparation of carbamate protected azabicycloalkenes $0.15 \mathrm{~g}(0.50 \mathrm{mmol})$ of bromide 6 was converted to $0.11 \mathrm{~g}$ of azabicycloalkene (3S)-8b using $10 \mathrm{Mol} \%(R)$-tolBinap and $\mathrm{CuClO}_{4} \cdot 4 \mathrm{MeCN}$ as catalyst $(79 \%) ; R_{\mathrm{f}}=0.45(\mathrm{PE} / \mathrm{EtOAc} 1: 1 ; \mathrm{UV}) ;{ }^{1} \mathrm{H}-\mathrm{NMR}\left(500 \mathrm{MHz}, \mathrm{CDCl}_{3}\right.$, mixture of rotamers): $\delta 7.35-7.26(\mathrm{~m}, 5 \mathrm{H}), 6.52(\mathrm{~m}, 0.5 \mathrm{H}), 6.39(\mathrm{~m}, 1.5 \mathrm{H}), 5.01-5.17(\mathrm{~m}, 2 \mathrm{H})$, 4.88 (br s, 0.5H), 4.79 (br s, $0.5 \mathrm{H}), 3.78(\mathrm{~s}, 1.5 \mathrm{H}), 3.68(\mathrm{~s}, 1.5 \mathrm{H}), 3.58$ (br s, 0.5H), $3.54(\mathrm{br} \mathrm{s}, 0.5 \mathrm{H}), 3.34(\mathrm{~s}, 1 \mathrm{H}), 2.01(\mathrm{~d}, 1 \mathrm{H}, \mathrm{J}=8.6 \mathrm{~Hz}), 1.51(\mathrm{~d}, 1 \mathrm{H}, \mathrm{J}=8.6 \mathrm{~Hz}) ;{ }^{13} \mathrm{C}-$ NMR $\left(\mathrm{CDCl}_{3}, 101 \mathrm{MHz}\right.$, mixture of rotamers): $\delta 172.1,137.7,137.4,136.9,128.8$, 128.3, 128.2, 127.8, 67.5, 67.2, 62.1, 61.5, 59.5, 59.1, 52.8, 49.4, 48.6, 46.1, 45.6; HRMS (ESI): calcd. for $\mathrm{C}_{16} \mathrm{H}_{17} \mathrm{NO}_{4}\left(\mathrm{MNa}^{+}\right)$: 310.1055; found: 310.1045 ; HPLC (CHIRALPAK $^{\circledR}$ AD-H $(5 \mu \mathrm{m})$, Hexan: iso-Propanol $80: 20$, flow $0.5 \mathrm{ml} / \mathrm{min}, \lambda=$ $210 \mathrm{~nm})$ : ee $=72 \% ; t_{\mathrm{R}}($ major $)=16.6 \mathrm{~min} ; t_{\mathrm{R}}(\operatorname{minor})=19.1 \mathrm{~min}$. 

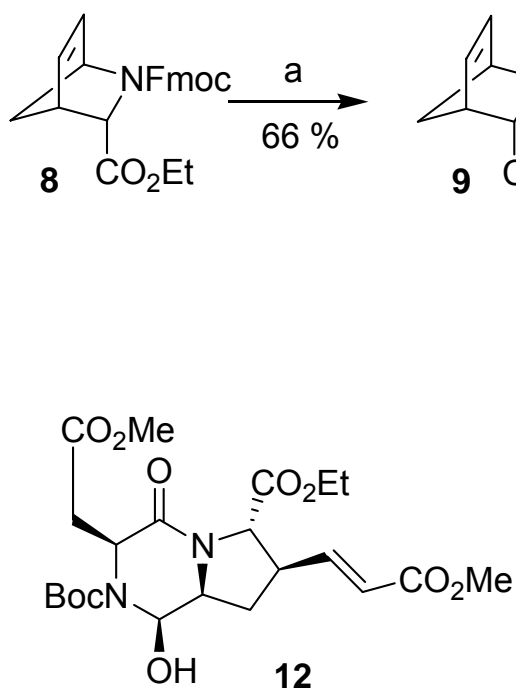
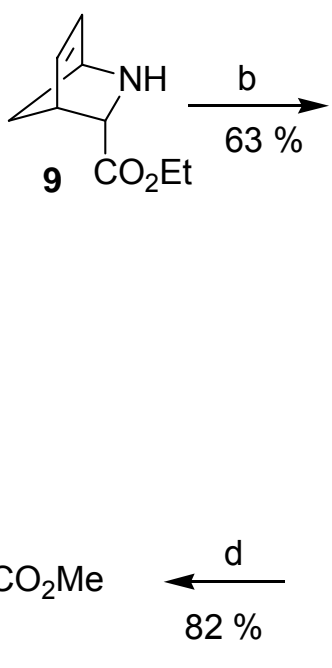<smiles>CCCCNC(CC(=O)OCC)C(=O)N1C2C=CC1C2COC(C)=O</smiles>

10, $\mathrm{dr}>95: 5$

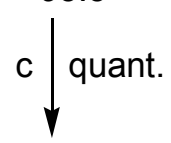<smiles>CCOC(=O)[C@@H]1[C@@H](C=O)C[C@H]2[C@@H](O)N(C(=O)OC(C)(C)C)[C@H](CC(=O)OC)C(=O)N12</smiles>

Reagents and conditions: ${ }^{a}$ piperidine, DMF, $1 \mathrm{~h}, \mathrm{rt} ;{ }^{\mathrm{b}} \mathrm{DCC}, \mathrm{HOBt}, \mathrm{Boc}-\mathrm{Asp}(\mathrm{OMe})-\mathrm{OH}, \mathrm{CH}_{2} \mathrm{Cl}_{2}, 0{ }^{\circ} \mathrm{C}$, $16 \mathrm{~h} ;{ }^{\mathrm{c}} \mathrm{O}_{3}, \mathrm{CH}_{2} \mathrm{Cl}_{2},-78{ }^{\circ} \mathrm{C}$; then dimethylsulfide, $\mathrm{CH}_{2} \mathrm{Cl}_{2}, 1 \mathrm{~h}$, rt; ${ }^{d} \mathrm{Ph}_{3} \mathrm{P}=\mathrm{CHCO}_{2} \mathrm{Me}$, THF, rt, $18 \mathrm{~h}$.

\section{Azabicycloalkene 9}

Under nitrogen atmosphere, $150 \mathrm{mg}(0.40 \mathrm{mmol})$ of azabicycloalkene (3S)-8 (prepared as described above for (3S)-8a in $76 \%$ yield, $66 \%$ ee) was added to 10 $\mathrm{ml}$ of a $20 \%$ solution of piperidine in DMF. The resulting solution was stirred for $2 \mathrm{~h}$ at room temperature. After evaporation of the solvent, the product was purified by column chromatography using PE / EtOAc 2:1 $\rightarrow \mathrm{CH}_{2} \mathrm{Cl}_{2} / \mathrm{MeOH}$ 9:1 as eluent. Drying in vacuo yielded $49 \mathrm{mg}(0.31 \mathrm{mmol})$ of a colourless wax $(78 \%) . R_{\mathrm{f}}=0.2(\mathrm{PE} /$ EtOAc, 2:1; Mo/Ce); ${ }^{1} \mathrm{H}-\mathrm{NMR}\left(500 \mathrm{MHz}, \mathrm{CDCl}_{3}\right): \delta 6.11-6.14(\mathrm{~m}, 2 \mathrm{H}), 4.05$ (q, $2 \mathrm{H}, \mathrm{J}$ = $7.1 \mathrm{~Hz}), 3.94(\mathrm{br} \mathrm{s}, 1 \mathrm{H}), 3.12(\mathrm{br}, 1 \mathrm{H}), 2.80(\mathrm{br}, 1 \mathrm{H}), 2.08(\mathrm{br}, 1 \mathrm{H}), 1.47-1.50(\mathrm{~m}$, $1 \mathrm{H}), 1.20-1.24(\mathrm{~m}, 1 \mathrm{H}), 1.14(\mathrm{t}, 3 \mathrm{H}, \mathrm{J}=7.1 \mathrm{~Hz}) ;{ }^{13} \mathrm{C}-\mathrm{NMR}\left(101 \mathrm{MHz}, \mathrm{CDCl}_{3}\right): \delta$ $174.9,137.0,136.3,61.4,60.8,58.0,48.5,46.0,14.6$.

\section{Azabicycloalkene 10}

A solution of $95 \mathrm{mg}(0.39 \mathrm{mmol})$ Boc-Asp $(\mathrm{OMe})-\mathrm{OH}$ and $35 \mathrm{mg}(0.26 \mathrm{mmol}) \mathrm{HOBt}$ in $2 \mathrm{ml}$ dry DMF was added to a solution of $43 \mathrm{mg}(0.26 \mathrm{mmol})$ of azabicycloalkene 9 in $1 \mathrm{ml} \mathrm{DMF}$. After cooling to $0{ }^{\circ} \mathrm{C} 80 \mathrm{mg}(0.39 \mathrm{mmol}) \mathrm{DCC}$ was added and the reaction was stirred under a nitrogen atmosphere at room temperature for $20 \mathrm{~h}$. The solid precipitate was filtered off and the solvent was removed. The residue was dissolved in $5 \mathrm{ml}$ ethyl acetate and washed with each $5 \mathrm{ml}$ aqueous $\mathrm{KHSO}_{4}-\mathrm{NaHCO}_{3}$ - and $\mathrm{NaCl}$-solution. The organic layer was dried over $\mathrm{NaSO}_{4}$, filtered and the solvent was removed in vacuo. The product was purified by column chromatography using PE / EtOAc 3:1 $\rightarrow$ PE / EtOAc 2:1 as eluent. Drying in vacuo yielded $65 \mathrm{mg}$ of a 
colourless wax $(63 \%) . R_{\mathrm{f}}=0.41(\mathrm{PE} / \mathrm{EtOAc} 3: 1 ; \mathrm{Mo} / \mathrm{Ce}) ;{ }^{1} \mathrm{H}-\mathrm{NMR}(500 \mathrm{MHz}$, $\mathrm{CDCl}_{3}, 7: 3$ mixture of rotamers): $\delta 6.35-6.54(\mathrm{~m}, 2 \mathrm{H}), 5.49(\mathrm{~d}, 0.3 \mathrm{H}, J=9.5 \mathrm{~Hz})$, $5.33(\mathrm{~d}, 0.7 \mathrm{H}, \mathrm{J}=9.5 \mathrm{~Hz}), 4.83-5.20(\mathrm{~m}, 1.85 \mathrm{H}), 4.35(\mathrm{~m}, 0.15 \mathrm{H}), 4.12-4.28(\mathrm{~m}$, $2 \mathrm{H}), 3.54-3.71(\mathrm{~m}, 4 \mathrm{H}), 3.41-3.50(\mathrm{~m}, 0.3 \mathrm{H}), 3.29(\mathrm{~s}, 0.7 \mathrm{H}), 2.66-2.83(\mathrm{~m}, 1.4 \mathrm{H})$, $2.50-2.62(\mathrm{~m}, 0.6 \mathrm{H}), 2.06-2.10(\mathrm{~m}, 0.5 \mathrm{H}), 1.58-1.70(\mathrm{~m}, 1.5 \mathrm{H}), 1.43(\mathrm{~s}, 9 \mathrm{H}), 1.20$ - $1.29(\mathrm{~m}, 3 \mathrm{H}) ;{ }^{13} \mathrm{C}-\mathrm{NMR}\left(101 \mathrm{MHz}, \mathrm{CDCl}_{3}\right.$, mixture of rotamers): $\delta 171.7,171.1$, 168.8, 138.2, 138.0, 137.3, 136.6, 136.2, 80.3, 62.7, 62.4, 61.4, 60.9, 59.3, 58.5, 58.4, 52.0, 52.0, 49.2, 49.0, 48.7, 47.5, 47.5, 46.1, 45.9, 44.5, 37.4, 37.2, 34.0, 31.0, 14.2; HRMS (ESI): calcd. for $\mathrm{C}_{19} \mathrm{H}_{28} \mathrm{~N}_{2} \mathrm{O}_{7}\left(\mathrm{MH}^{+}\right)$: 397.1975 ; found: 397.1987 .

\section{Diazabicycloalkane 12}

$65 \mathrm{mg}(0.16 \mathrm{mmol})$ of azabicycloalkene 10 was dissolved in $6 \mathrm{ml} \mathrm{CH}_{2} \mathrm{Cl}_{2}$ and cooled to $-78{ }^{\circ} \mathrm{C}$. Ozone was bubbled through the solution until a blue colour persisted. After nitrogen was bubbled through the solution for a short time, $1 \mathrm{ml}$ dimethylsulfide was added. The solution was stirred for $30 \mathrm{~min}$ at $-78^{\circ} \mathrm{C}$ and the solvent was evaporated to give diazabicycloalkane $\mathbf{1 1}$ in quantitative yield. The residue was dissolved in abs. THF and $110 \mathrm{mg}(0.33 \mathrm{mmol}) \mathrm{Ph}_{3} \mathrm{P}=\mathrm{CHCO}_{2} \mathrm{Me}$ was added. The reaction was stirred for $18 \mathrm{~h}$ under an atmosphere of nitrogen. The product was purified by column chromatography (PE / EtOAc 10:1). Drying in vacuo yielded $64 \mathrm{mg}$ of diazabicycloalkane 12 (82\%). Analytical data were identical with those previously reported by us. ${ }^{4}$

\footnotetext{
${ }^{4}$ Maison, W.; Grohs, D. C.; Prenzel, A. H. G. P. Eur. J. Org. Chem. 2004, 1527-1543.
} 


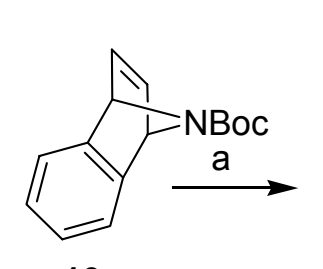

16



17

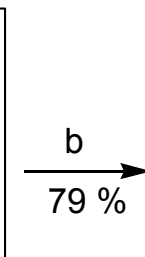

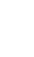

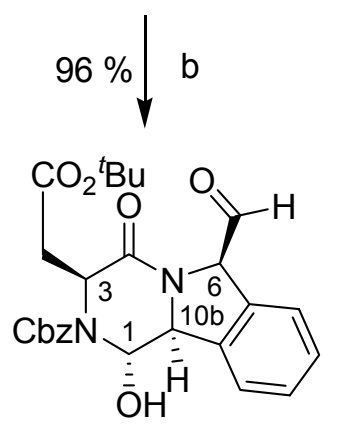

19, $d r>95: 5$

Reagents and conditions: ${ }^{a} \mathrm{AcCl}, \mathrm{MeOH}, 0{ }^{\circ} \mathrm{C}, 2 \mathrm{~h}$; then $\mathrm{Cbz}-\mathrm{Asp}\left(\mathrm{O}^{\mathrm{t}} \mathrm{Bu}\right)-\mathrm{OH}, \mathrm{DCC}, \mathrm{HOBt}, \mathrm{CH}_{2} \mathrm{Cl}_{2}, 12$ $\mathrm{h}, \mathrm{rt} ;{ }^{\mathrm{b}} \mathrm{OsO}_{4}, \mathrm{NaIO}_{4}, \mathrm{THF}, \mathrm{rt}, 8 \mathrm{~h} ;{ }^{\mathrm{c}} \mathrm{Ph}_{3} \mathrm{P}=\mathrm{CHCO}_{2} \mathrm{Me}, \mathrm{THF}, 5 \mathrm{~h}$, rt.

\section{Alkene 18}

To $50 \mathrm{ml}$ abs. $\mathrm{MeOH}$ was added dropwise $5.9 \mathrm{ml}$ freshly distilled acetyl chloride (82 $\mathrm{mmol}$ ) at $0{ }^{\circ} \mathrm{C}$ under a nitrogen atmosphere. The resulting solution was stirred for 5 min at $0{ }^{\circ} \mathrm{C}$ and $3.5 \mathrm{~g}$ azabicycloalkene $16(14.4 \mathrm{mmol})$ were added. The solution was allowed to reach room temperature and stirred for additional $2 \mathrm{~h}$. Water $(200 \mathrm{ml})$ was added and the mixture was extracted with diethylether two times. The aqueous layer was subsequently adjusted to $\mathrm{pH} \sim 10$ with solid potassium carbonate and extracted three times with each $100 \mathrm{ml}$ diethylether. The combined extracts were dried over $\mathrm{Na}_{2} \mathrm{SO}_{4}$ and the solvent was removed in vacuo ( $400 \mathrm{mbar}$ ) to give free amine 17 with some residual solvent. $R_{f}=0.41\left(\mathrm{CH}_{2} \mathrm{Cl}_{2} / \mathrm{MeOH} 9: 1\right) ;{ }^{1} \mathrm{H}-\mathrm{NMR}$ $\left(\mathrm{CDCl}_{3}, 400 \mathrm{MHz}\right): \delta$ 7.24-7.19 (m, $\left.2 \mathrm{H}\right)$, 7.01-6.99 (m, $\left.2 \mathrm{H}\right), 6.93-6.90(\mathrm{~m}, 2 \mathrm{H}), 4.97$ (dd, $\left.{ }^{3} \mathrm{~J}=1.3 \mathrm{~Hz}, 2 \mathrm{H}\right), 2.38$ (bs, $\left.1 \mathrm{H}\right) ;{ }^{13} \mathrm{C}-\mathrm{NMR}\left(\mathrm{CDCl}_{3}, 100 \mathrm{MHz}\right): \delta 151.2,144.5$, 124.7, 120.8, 66.3.

This crude product was dissolved in abs. dichloromethane and $5.1 \mathrm{~g} \mathrm{Cbz-Asp}\left(\mathrm{O}^{t} \mathrm{Bu}\right)-$ $\mathrm{OH}(15.8 \mathrm{mmol}), 3.3 \mathrm{~g} \mathrm{DCC}(15.8 \mathrm{mmol})$ and $2.1 \mathrm{~g} \mathrm{HOBt}(15.8 \mathrm{mmol})$ were added. The resulting suspension was strirred under nitrogen atmosphere at it for $12 \mathrm{~h}$, filtered and evaporated. The residue was treated with $100 \mathrm{ml}$ ethyl acetate and washed with each $100 \mathrm{ml}$ aqueous saturated $\mathrm{KHSO}_{4}(2 \mathrm{x})$ and $100 \mathrm{ml}$ aqueous saturated $\mathrm{NaHCO}_{3}(2 \mathrm{x})$. The organic phase was dried over $\mathrm{Na}_{2} \mathrm{SO}_{4}$, filtered and the solvent removed in vacuo to give $6.4 \mathrm{~g}$ of a yellow oil. Chromatography on silica (gradient PE / EtOAc 7:3 - 1:1) gave $5.10 \mathrm{~g}$ of pure alkene 18 (79 \%) as a colourless 
oil; $R_{f}=0.48$ (PE / EtOAc, 7:3); ${ }^{1} \mathrm{H}-\mathrm{NMR}\left(\mathrm{CDCl}_{3}, 500 \mathrm{MHz}\right.$, some signals are broadened or doubled due to rotational isomerism): $\delta 1.32(\mathrm{~s}, 4.5 \mathrm{H}), 1.42(\mathrm{~s}, 4.5 \mathrm{H})$, $2.44\left(\mathrm{dd}, 0.5 \mathrm{H},{ }^{2} \mathrm{~J}=15.8 \mathrm{~Hz},{ }^{3} \mathrm{~J}=6.3 \mathrm{~Hz}\right), 2.51$ (dd, $0.5 \mathrm{H},{ }^{2} \mathrm{~J}=5.7 \mathrm{~Hz},{ }^{3} \mathrm{~J}=5.7 \mathrm{~Hz}$ ), $2.54\left(\mathrm{dd}, 0.5 \mathrm{H},{ }^{2} \mathrm{~J}=5.7 \mathrm{~Hz},{ }^{3} \mathrm{~J}=5.7 \mathrm{~Hz}\right), 2.65\left(\mathrm{dd}, 0.5 \mathrm{H},{ }^{2} \mathrm{~J}=15.8 \mathrm{~Hz},{ }^{3} \mathrm{~J}=6.6 \mathrm{~Hz}\right)$, $4.77-4.83(\mathrm{~m}, 1 \mathrm{H}), 5.05\left(\mathrm{~d}, 0.5 \mathrm{H},{ }^{2} \mathrm{~J}=12.3 \mathrm{~Hz}\right), 5.10\left(\mathrm{~d}, 0.5 \mathrm{H},{ }^{2} \mathrm{~J}=5.7 \mathrm{~Hz}\right), 5.12(\mathrm{~d}$, $\left.0.5 \mathrm{H},{ }^{2} \mathrm{~J}=5.7 \mathrm{~Hz}\right), 5.16\left(\mathrm{~d}, 0.5 \mathrm{H},{ }^{2} \mathrm{~J}=12.3 \mathrm{~Hz}\right), 5.46\left(\mathrm{~d}, 0.5 \mathrm{H},{ }^{3} \mathrm{~J}=9.5 \mathrm{~Hz}\right), 5.68(\mathrm{~d}$, $\left.0.5 \mathrm{H},{ }^{3} \mathrm{~J}=9.1 \mathrm{~Hz}\right), 5.85(\mathrm{~s}, 1 \mathrm{H}), 5.93\left(\mathrm{~d}, 1 \mathrm{H},{ }^{3} \mathrm{~J}=3.8 \mathrm{~Hz}\right), 6.81\left(\mathrm{~d}, 0.5 \mathrm{H},{ }^{3} \mathrm{~J}=3.1 \mathrm{~Hz}\right)$, 6.87-7.01 (m, 3.5H), $7.08\left(\mathrm{~d}, 0.5 \mathrm{H},{ }^{3} \mathrm{~J}=6.6 \mathrm{~Hz}\right), 7.24-7.36(\mathrm{~m}, 6.5 \mathrm{H}) ;{ }^{13} \mathrm{C}-\mathrm{NMR}$ $\left(\mathrm{CDCl}_{3}, 125 \mathrm{MHz}\right.$, some signals are broadened or doubled due to rotational isomerism): $\delta$ 28.0, 28.1, 38.2, 48.9, 49.0, 63.4, 63.5, 65.4, 65.5, 67.1, 67.2, 81.4, $120.7,120.8,121.3,121.4,125.3,125.5,128.2,128.3,128.6,136.4,136.5,142.8$, 143.0, 143.3, 144.2, 147.5, 147.9, 148.0, 148.1, 155.5, 155.7, 164.0, 169.6, 169.8; Anal. calcd. for $\mathrm{C}_{26} \mathrm{H}_{28} \mathrm{~N}_{2} \mathrm{O}_{5}$ : C, 69.63; $\mathrm{H}, 6.29 ; \mathrm{N}, 6.25$; found: $\mathrm{C}, 69.70 ; \mathrm{H}, 6.25 ; \mathrm{N}$, 6.19 .

\section{Diazabicycloalkene 20}

A solution of $20 \mathrm{mg} \mathrm{OsO}$ ( $0.08 \mathrm{mmol})$ in $2 \mathrm{ml}$ water was added to $0.5 \mathrm{~g}$ alkene 18 $(1.1 \mathrm{mmol})$ in $14 \mathrm{ml}$ THF. After ten minutes, $0.82 \mathrm{~g} \mathrm{NalO}_{4}(3.85 \mathrm{mmol})$ in $10 \mathrm{ml}$ water was added and the reaction was stirred for $8 \mathrm{~h}$ at $\mathrm{rt}$. Diethylether was added and the resulting mixture was washed with saturated aqueous $\mathrm{Na}_{2} \mathrm{SO}_{3}$ solution and water. The organic phase was dried over $\mathrm{Na}_{2} \mathrm{SO}_{4}$, filtered and the solvent removed in vacuo to give $0.54 \mathrm{~g}$ of a colourless solid. Chromatography on silica (PE / EtOAc, 6:4) gave $0.51 \mathrm{~g}$ of slightly impurified aldehyde 19 (96\%) as a colourless sticky solid that was used in the next step without additional purification; $R_{f}=0.37$ (PE / EtOAc, 6:4).

The aldehyde 19 (200 mg, $0.42 \mathrm{mmol}$ ) was dissolved in $10 \mathrm{ml}$ abs. THF and treated with $278 \mathrm{mg} \mathrm{Ph}{ }_{3} \mathrm{P}=\mathrm{CHCO}_{2} \mathrm{Me}(0.84 \mathrm{mmol})$. After stirring for $8 \mathrm{~h}$ at $\mathrm{rt}$ the solvent was removed in vacuo and the residue purified by column chromatography on silica (PE / EtOAc, 6:4). The title compound 20 was obtained as a colourless solid in $167 \mathrm{mg}$ yield (74\%); $R_{f}=0.40(\mathrm{PE} / \mathrm{EtOAc}, 6: 4) ;{ }^{1} \mathrm{H}-\mathrm{NMR}\left(\mathrm{CDCl}_{3}, 400 \mathrm{MHz}\right.$, some signals are broadened or doubled due to rotational isomerism): $\delta 1.41(\mathrm{~s}, 9 \mathrm{H}), 2.95(\mathrm{br}$, $0.5 \mathrm{H}), 3.27(\mathrm{br}, 0.5 \mathrm{H}), 3.28\left(\mathrm{dd}, 1 \mathrm{H},{ }^{2} \mathrm{~J}=18.1 \mathrm{~Hz},{ }^{3} \mathrm{~J}=4.3 \mathrm{~Hz}\right), 3.67(\mathrm{~s}, 3 \mathrm{H}), 4.51-$ $4.55(\mathrm{~m}, 1 \mathrm{H}), 4.97-5.05(\mathrm{~m}, 1 \mathrm{H}), 5.16-5.38(\mathrm{~m}, 2 \mathrm{H}), 5.65\left(\mathrm{~d}, 1 \mathrm{H},{ }^{3} \mathrm{~J}=8.7 \mathrm{~Hz}\right), 5.87$ (br, 0.5H), 6.05-6.16 (m, 1H), $6.20\left(\mathrm{~d}, 1 \mathrm{H},{ }^{3} \mathrm{~J}=15.7 \mathrm{~Hz}\right), 6.27(\mathrm{br}, 0.5 \mathrm{H}), 6.88(\mathrm{dd}$, $\left.1 \mathrm{H},{ }^{3} \mathrm{~J}=15.7 \mathrm{~Hz},{ }^{3} \mathrm{~J}=8.7 \mathrm{~Hz}\right), 7.17\left(\mathrm{~d}, 1 \mathrm{H},{ }^{3} \mathrm{~J}=7.9 \mathrm{~Hz}\right), 7.30-7.47(\mathrm{~m}, 9 \mathrm{H}) ;{ }^{13} \mathrm{C}-\mathrm{NMR}$ 
$\left(\mathrm{CDCl}_{3}, 101 \mathrm{MHz}\right.$, some signals are broadened due to rotational isomerism): $\delta 28.1$, $36.9,38.6,51.5,53.1,64.9,66.8,68.3,75.0,83.2,121.9,122.9,124.1,128.4,128.6$, $128.8,129.1,129.2,134.8,135.8,137.4,146.5,154.7,163.7,165.3,166.2$; HRMS (FAB): calcd. for $\mathrm{C}_{29} \mathrm{H}_{33} \mathrm{~N}_{2} \mathrm{O}_{8}\left(\mathrm{MH}^{+}\right)=537.2237$; found 537.2240 . 


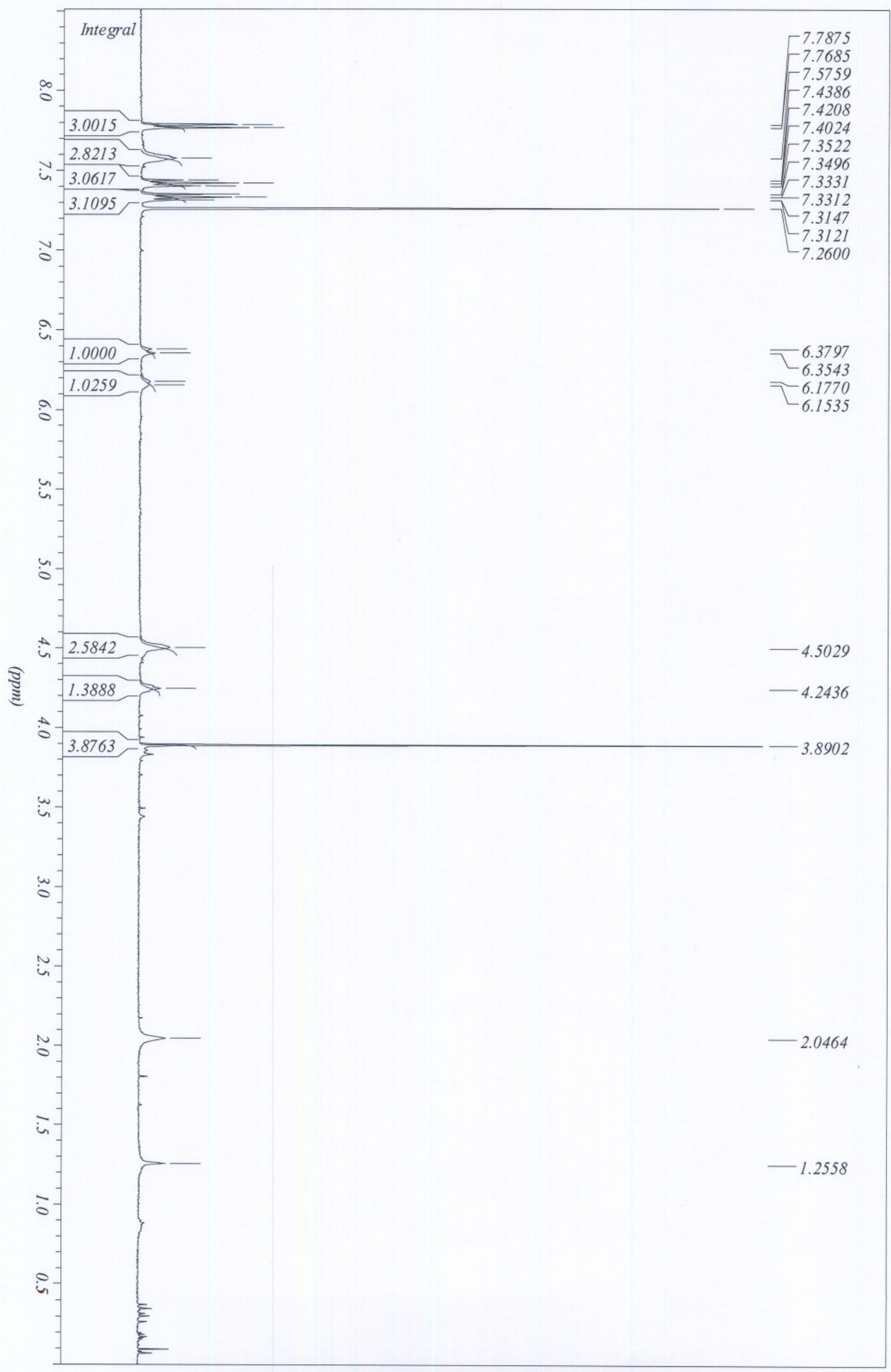




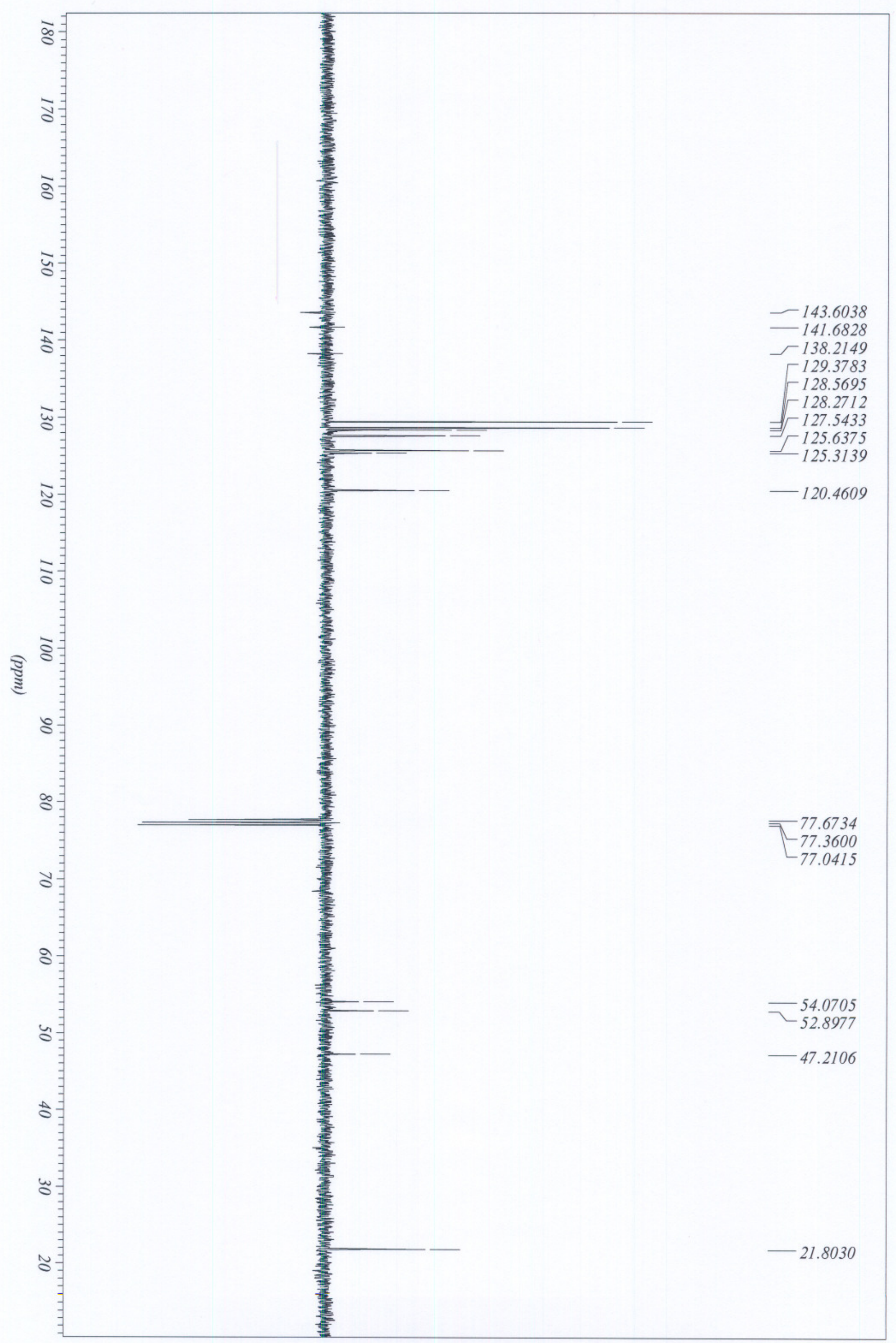




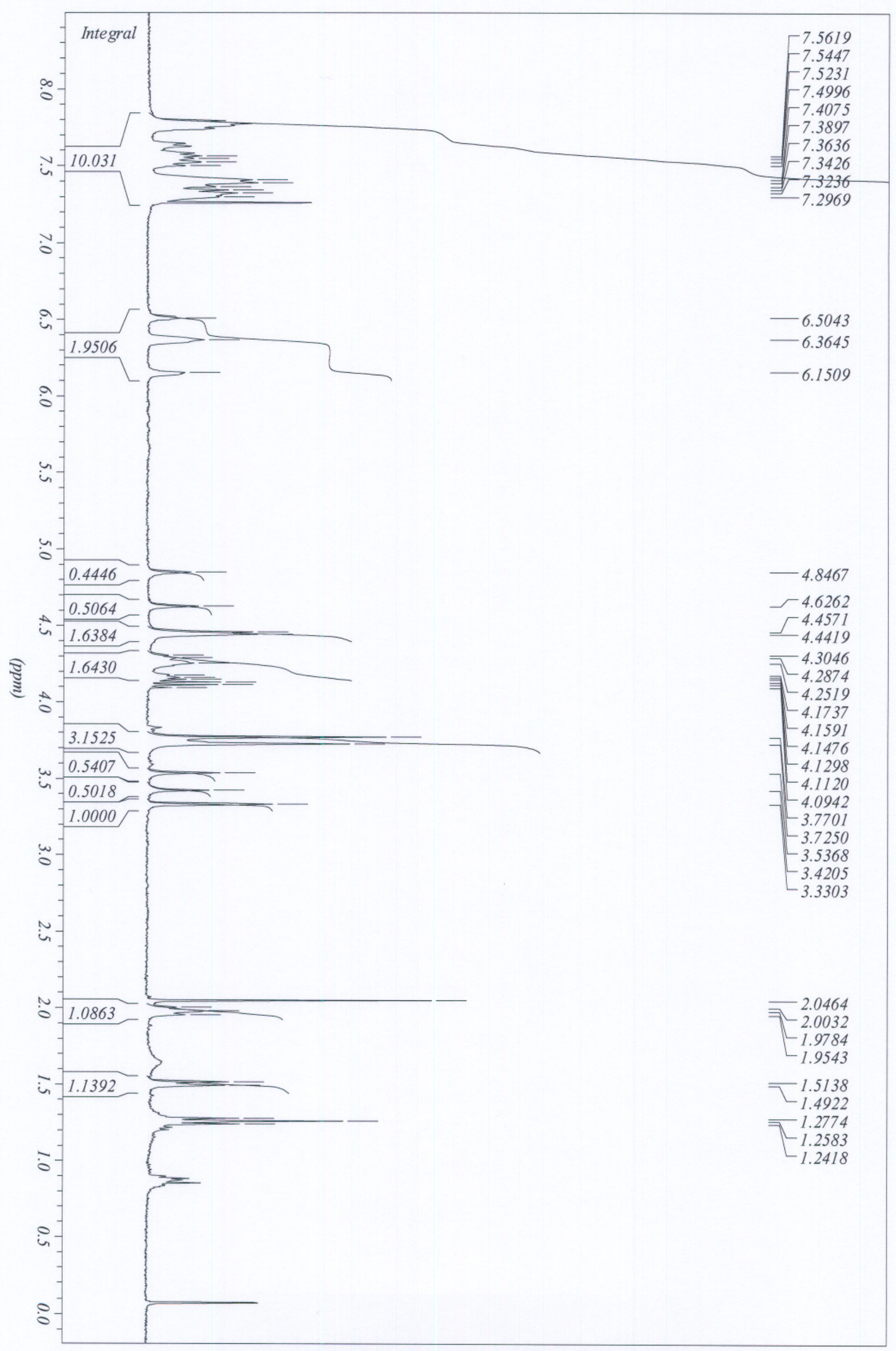




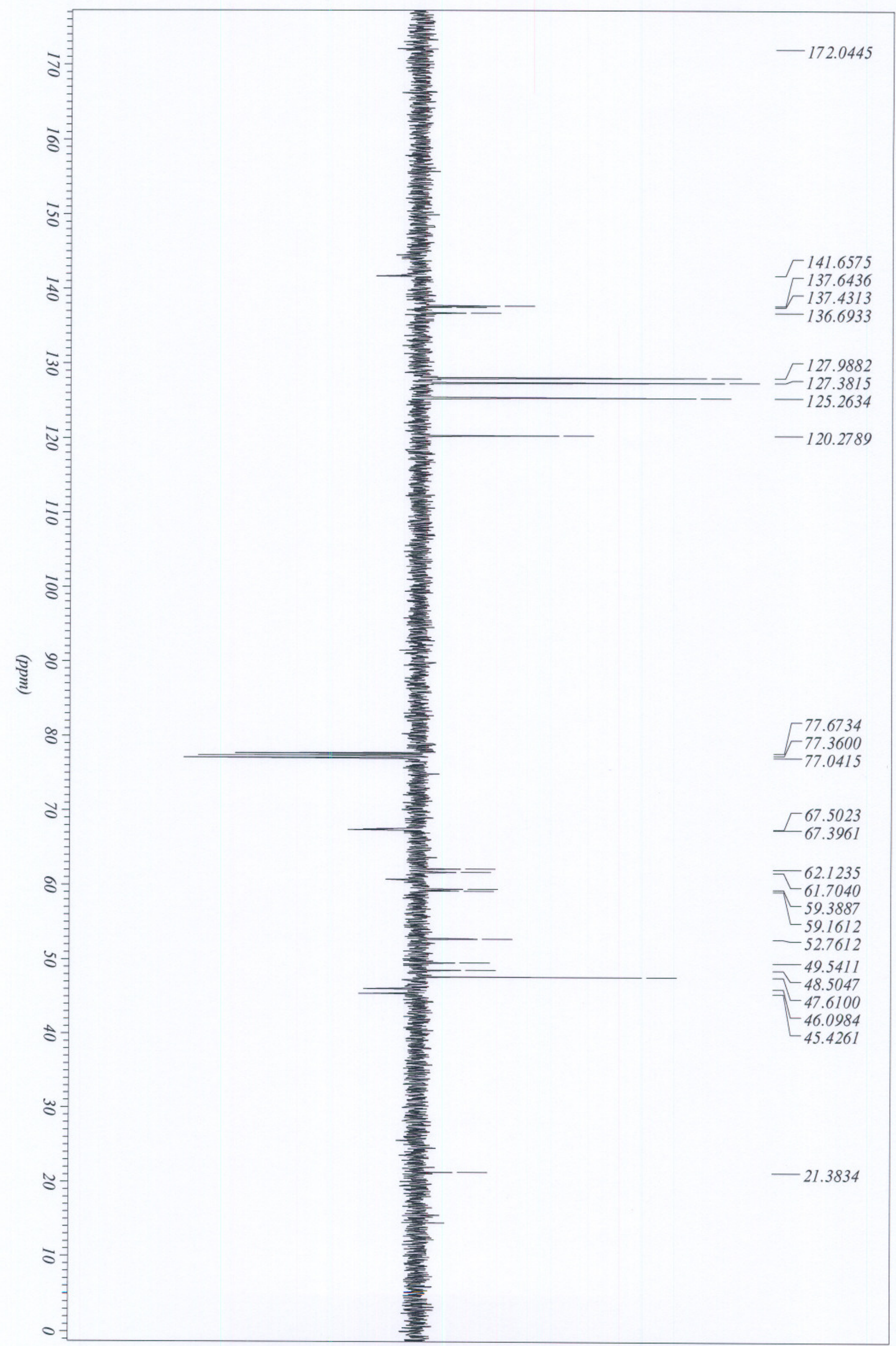




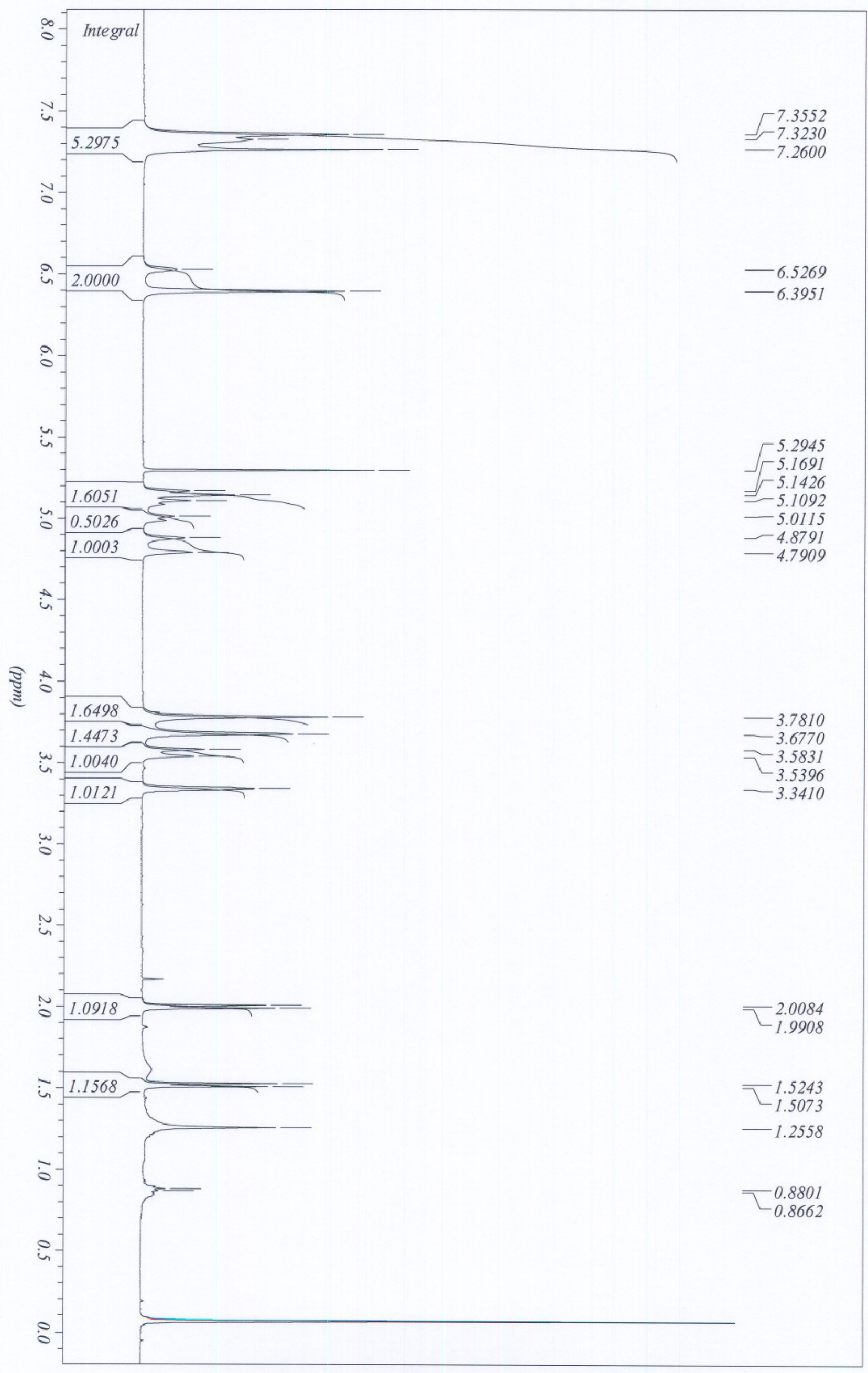














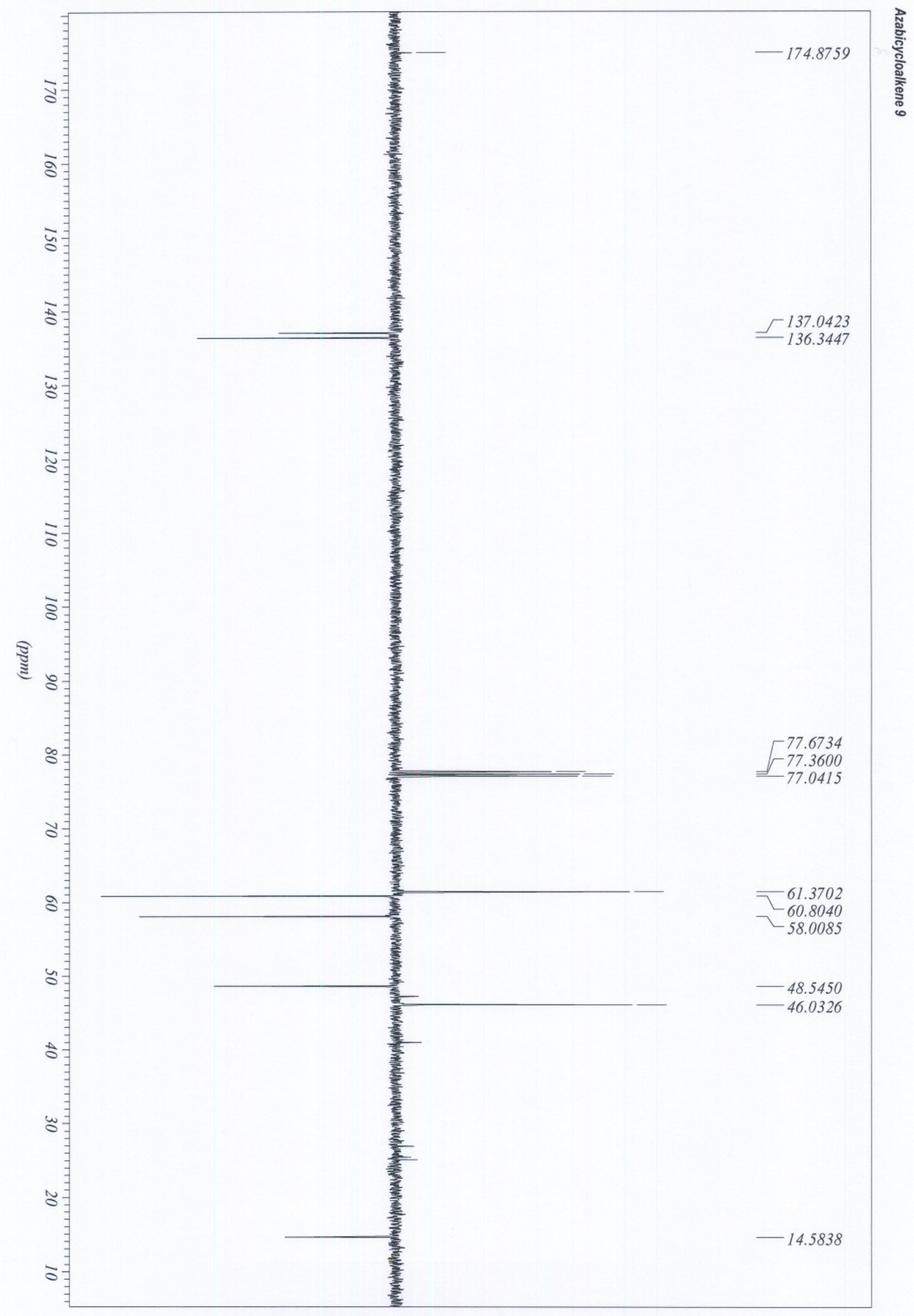




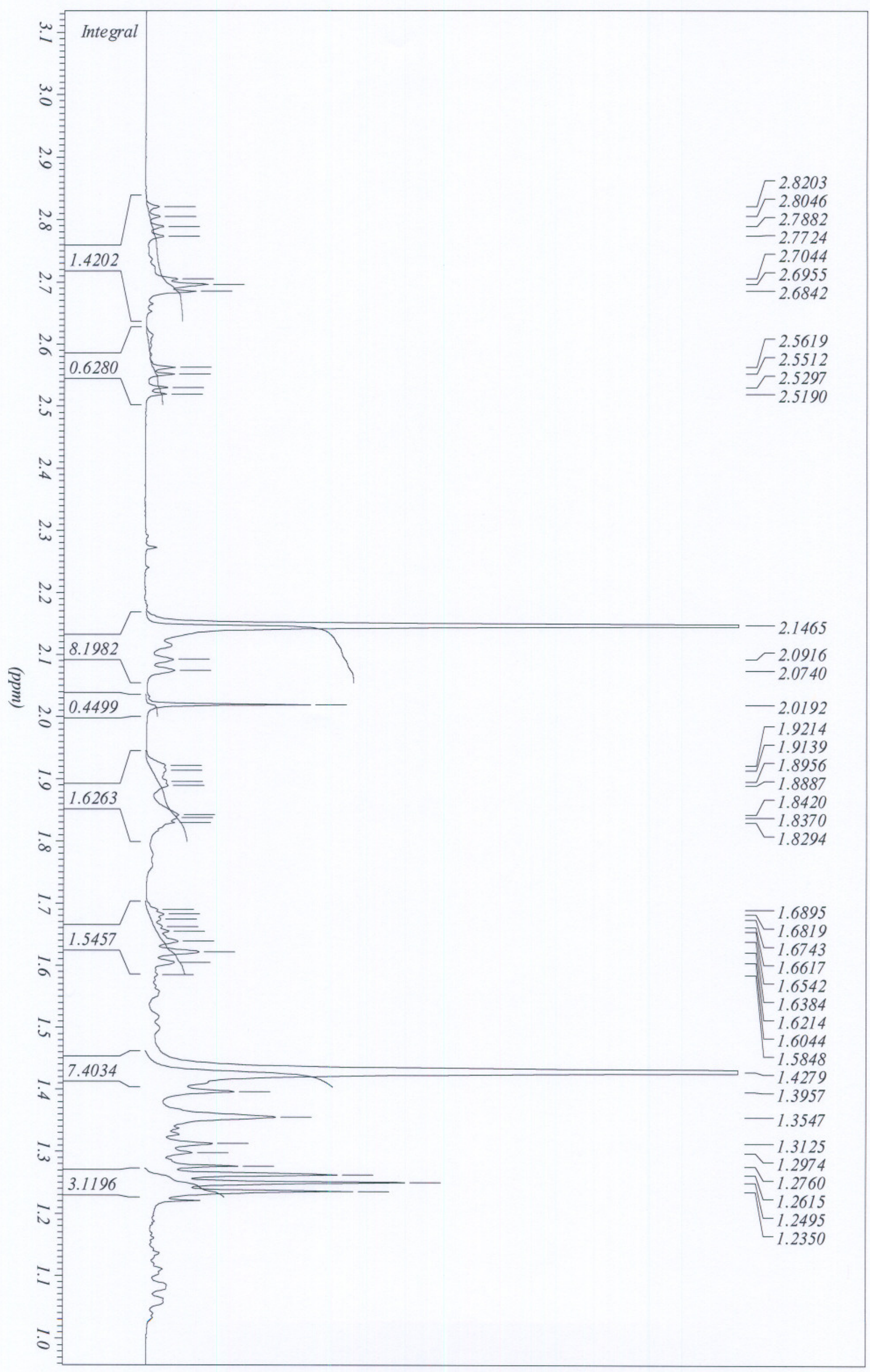




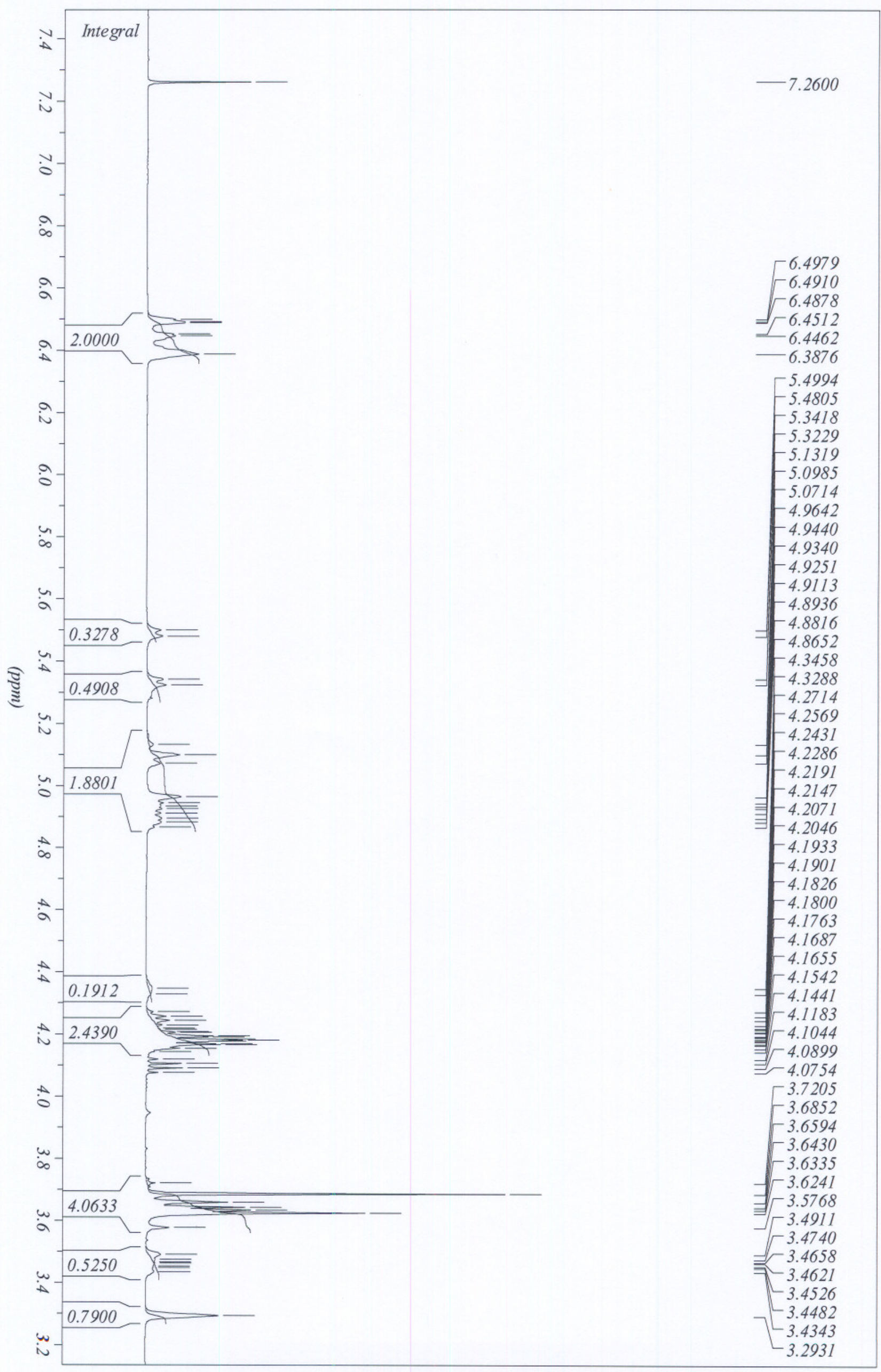









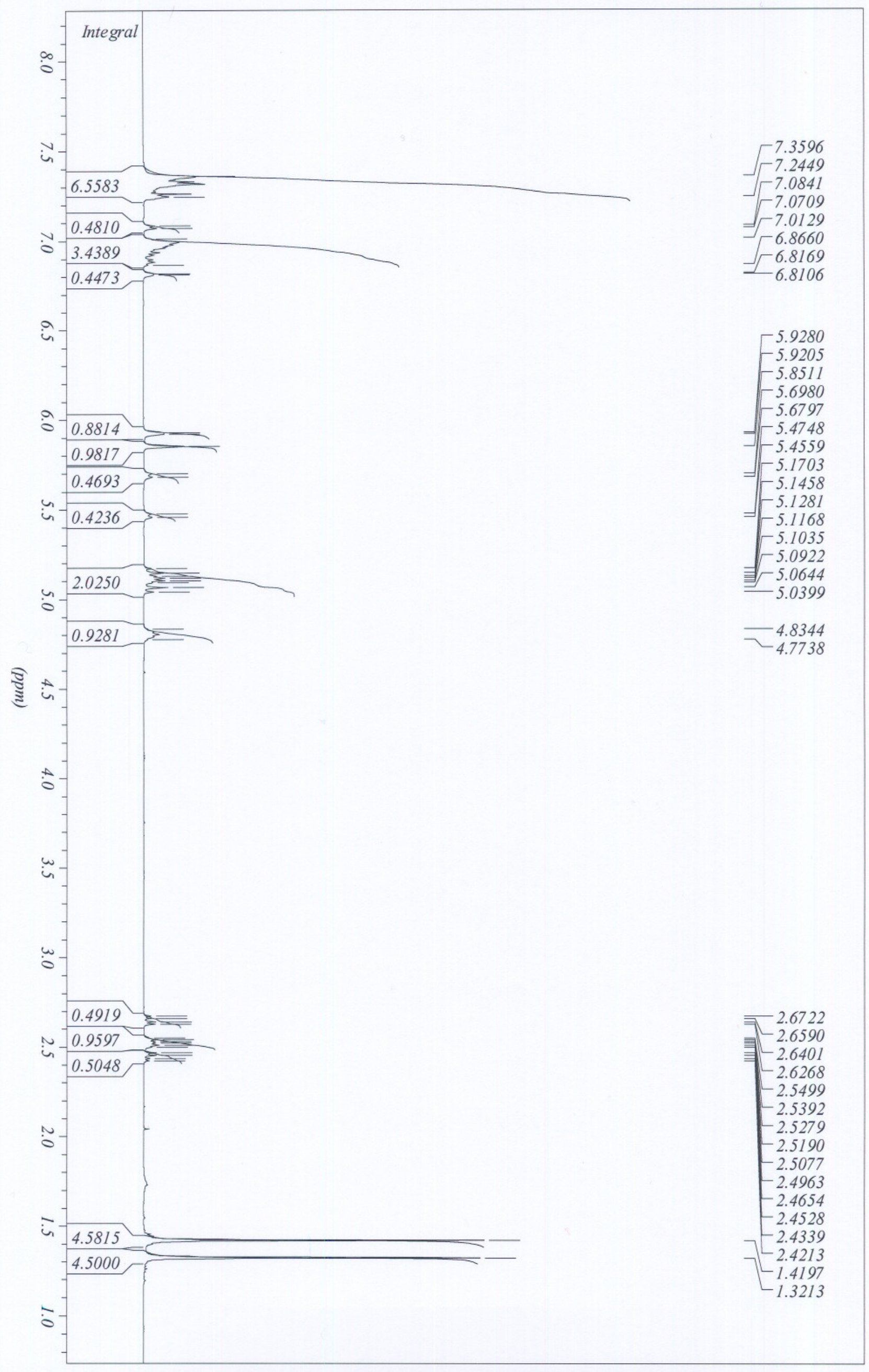




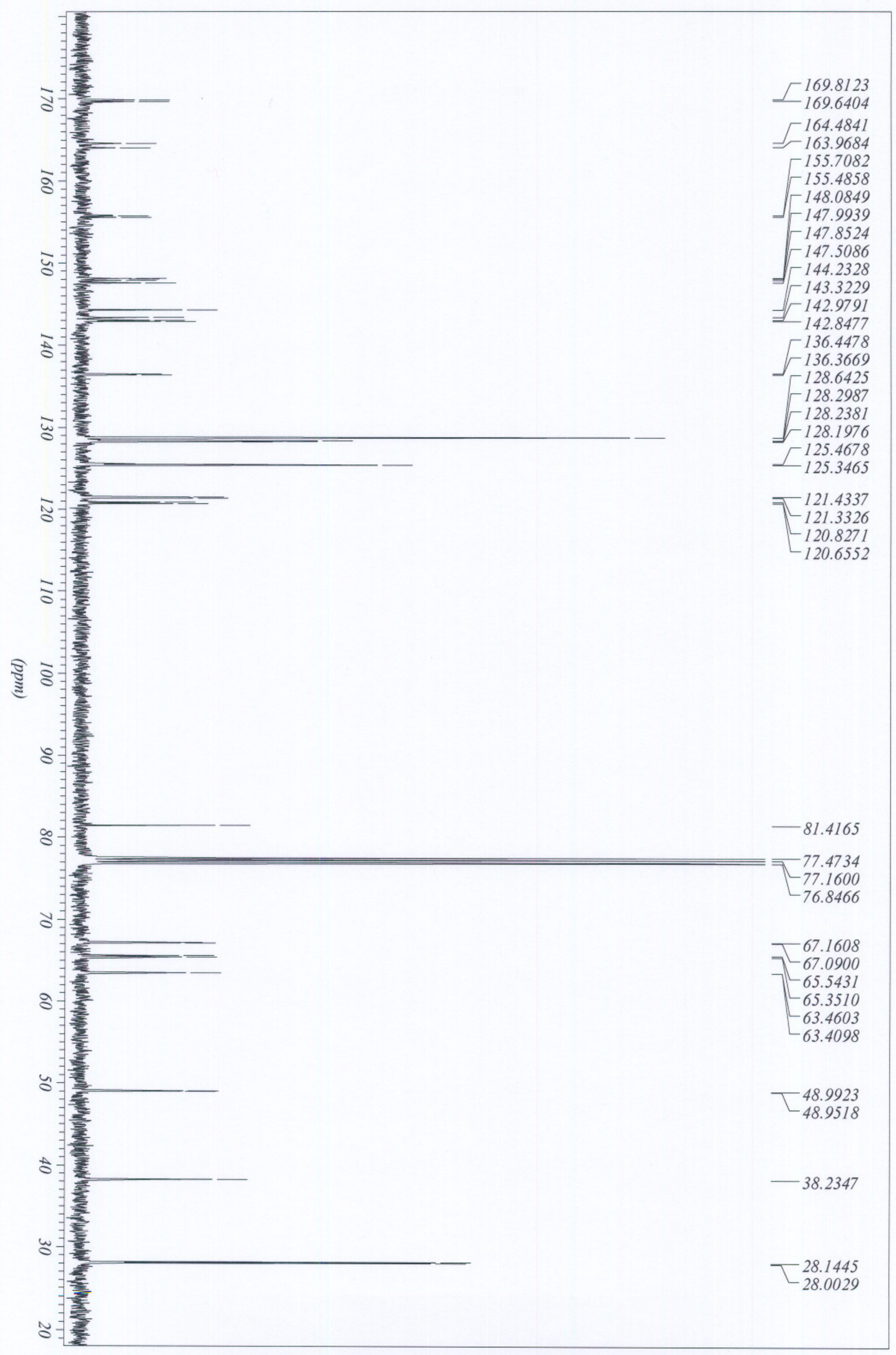

$-163.9684$

$-155.7082$

$-147.9939$

$-147.8524$

$-143.3229$

142.8477

$-136.4478$

$-136.3669$

$-128.6425$

$-128.2987$

$-128.2381$

$-128.1976$

125.3465

121.4337

$-121.3326$

$-120.8271$

$-81.4165$

77.1600
-76.8460

67.1608

67.0900

65.5431

65.3510

63.4603

63.4098

48.9923

48.9518

38.2347

28.1445

28.0029 


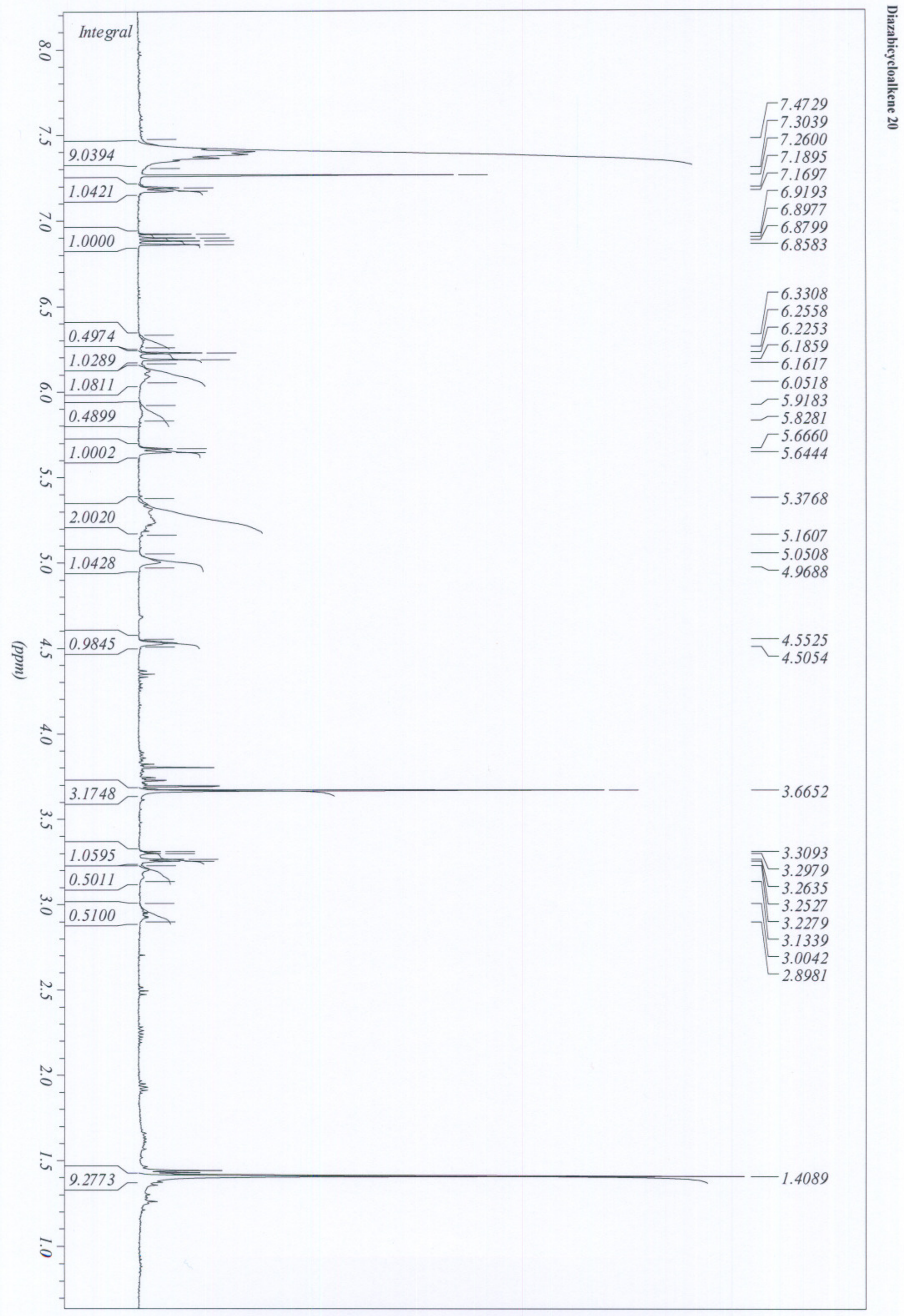




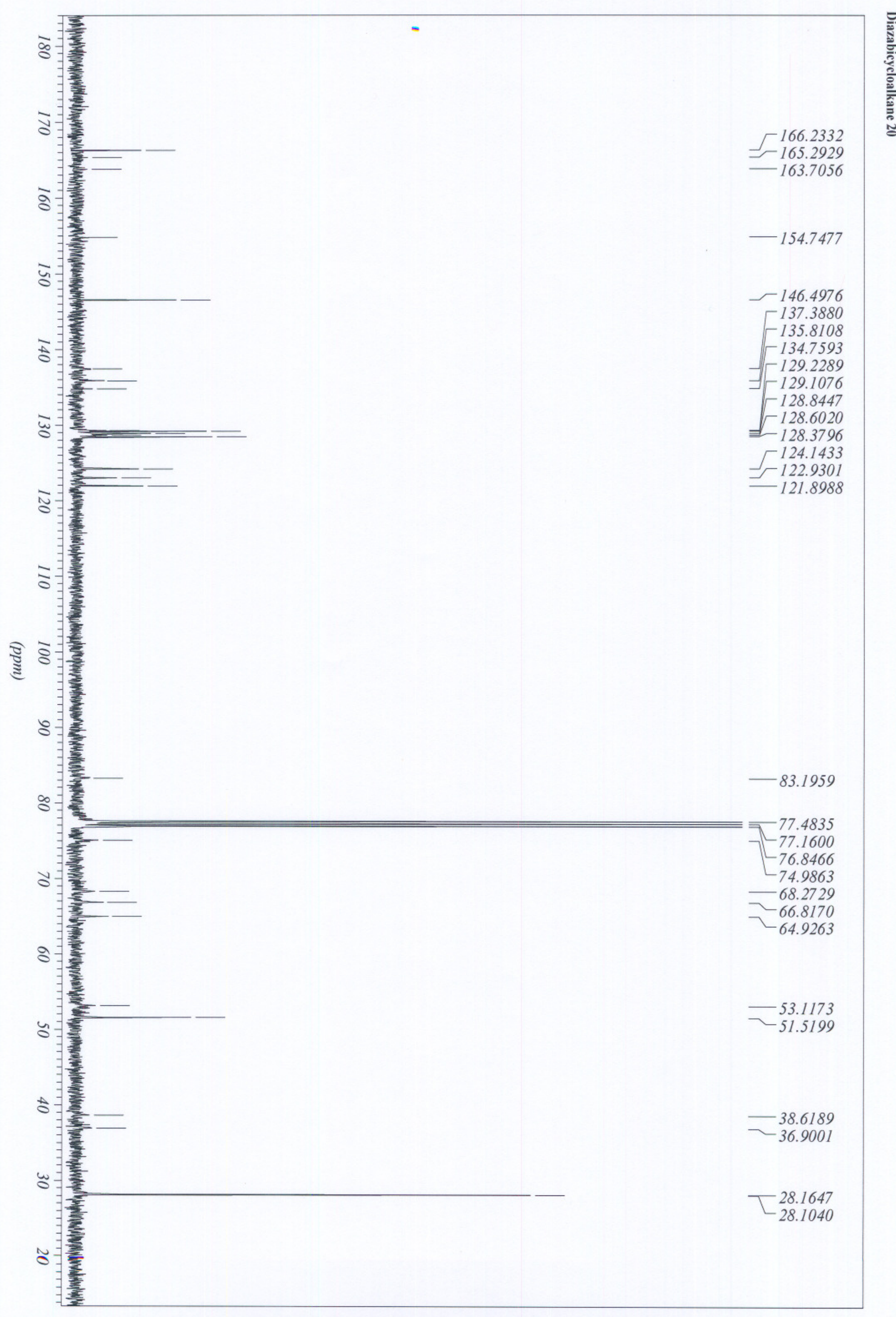

